\title{
Erratum to: Dynamic Analysis of a Fatigue Test Bench for High Precision Flexure Hinges
}

\author{
D. Schoenen, M. Hüsing and B. Corves
}

\section{Erratum to:}

Chapter "Dynamic Analysis of a Fatigue Test Bench for High Precision Flexure Hinges" in: L. Zentner et al. (eds.), Microactuators and Micromechanisms, Mechanisms and Machine Science 45, DOI 10.1007/978-3-319-45387-3_15

The author name in the original version of this chapter was published with incorrect initial as 'C. Corves'. It has now been updated with the correct initial as 'B. Corves'.

The updated original online version for this chapter can be found at 10.1007/978-3-319-45387-3_15

D. Schoenen $(\bowtie) \cdot$ M. Hüsing $\cdot$ B. Corves

Department of Mechanism Theory and Dynamics of Machines,

RWTH Aachen University, Aachen, Germany

e-mail: schoenen@igm.rwth-aachen.de

M. Hüsing

e-mail: huesing@igm.rwth-aachen.de

B. Corves

e-mail: corves@igm.rwth-aachen.de 